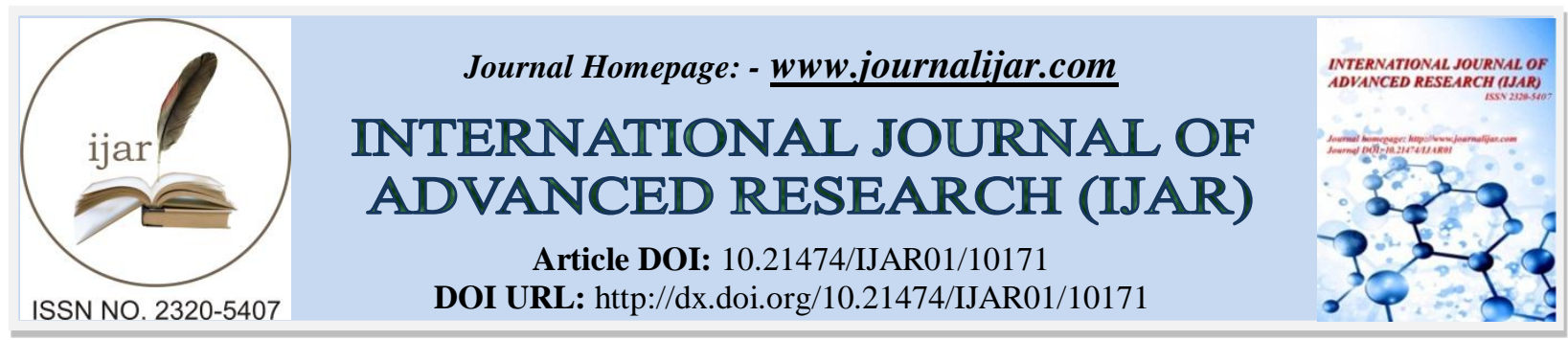

RESEARCH ARTICLE

\title{
LUNG INVOLVEMENT REVEALING MULTIPLE MYELOMA
}

\section{Y. Amchich ${ }^{1}$, N. Reguig ${ }^{2}$, A. Boucaid ${ }^{1}$, L. El akkari ${ }^{3}$, A. Zegmout ${ }^{1}$, H. Souhi ${ }^{1}$, N. Messaoudi ${ }^{3}$, H. El ouazzani ${ }^{1}$,} I.A. Rhorfi ${ }^{4}$ and A. Abid ${ }^{1}$

1. Department Of Pneumology, Military Hospital Mohamed V Rabat, Morocco.

2. Department Of Pneumology, Moulay Youssef University Hospital Center Rabat, Morocco.

3. Department Of Hematology, Military Hospital Mohamed V Rabat, Morocco.

4. Faculty Of Medicine and Pharmacy, Mohamed V University Rabat, Morocco.

\section{Manuscript Info}

.1..........................

Manuscript History

Received: 05 October 2019

Final Accepted: 07 November 2019

Published: December 2019

Key words:-

Multiple Myeloma, Interstitial Lung

Disease, Plasma Cells, Bronchoalveolar

Lavage

\section{Abstract}

Multiple Myeloma (MM) is a relatively rare disease and it presenting outside the bone marrow, known as extra-medullary myeloma (EMM), is rarer still. The lung parenchyma is an especially unusual site of involvement. We report a case in which the myeloma was diagnosed after we discovered the presence of monoclonal plasma cells in the bronchoalveolar lavage fluid (BAL). Analysis of BAL fluid for the presence of plasma cells provides a noninvasive means of establishing the diagnosis. Interstitial lung disease as pulmonary manifestation of multiple myeloma is even rarer; only isolated cases with histological proofs have been reported in the literature.

Copy Right, IJAR, 2019,. All rights reserved.

\section{Introduction:-}

Multiple Myeloma (MM) is a relatively rare disease, it is a malignant monoclonal gammopathy characterized by proliferation of myeloma cell clones responsible for their osteolytic lesion appearance (favoring pathological fractures and nerve compression), bone marrow infiltration (with the advent of bone marrow failure), abnormal protein production - M component (responsible for damage kidneys, the hyperviscosity syndrome and secondary amyloidosis), installing of immune deficiency (favoring infections).

The extramedullary myeloma (EMM) is present in $6 \%-8 \%$ of patients at the time of myeloma diagnosis [1]. Common sites of EMM involvement include hepatic, renal, pancreatic, pleural and the CNS. However, the pulmonary parenchyma is rarely involved [1,2]. One large study of 958 patients with MM found only 4 patients with presentations suggesting pulmonary infiltration by myeloma cells. Only one of these cases was proven histologically [2]. We report a very rare case of rapidly progressive pulmonary multiple myeloma.

\section{Case report:}

A 60-year-old man, smoker, with no history of respiratory hospitalization and relative good past health, was admitted in our hospital for chronic cough, significant weight loss and progressive dyspnea in the previous three months. Physical examination showed pale skin, without rales at pulmonary auscultation, pain in some small joints, blood pressure was normal but he had sinus tachycardia ( $100 \% / \mathrm{min}), \mathrm{SaO} 2=90 \%$.

Chest X-ray evidenced bilateral diffuse nodular opacities (Fig 1). Repeated exams of sputum smear were negative for Mycobacterium tuberculosis (both microscopy and culture). 


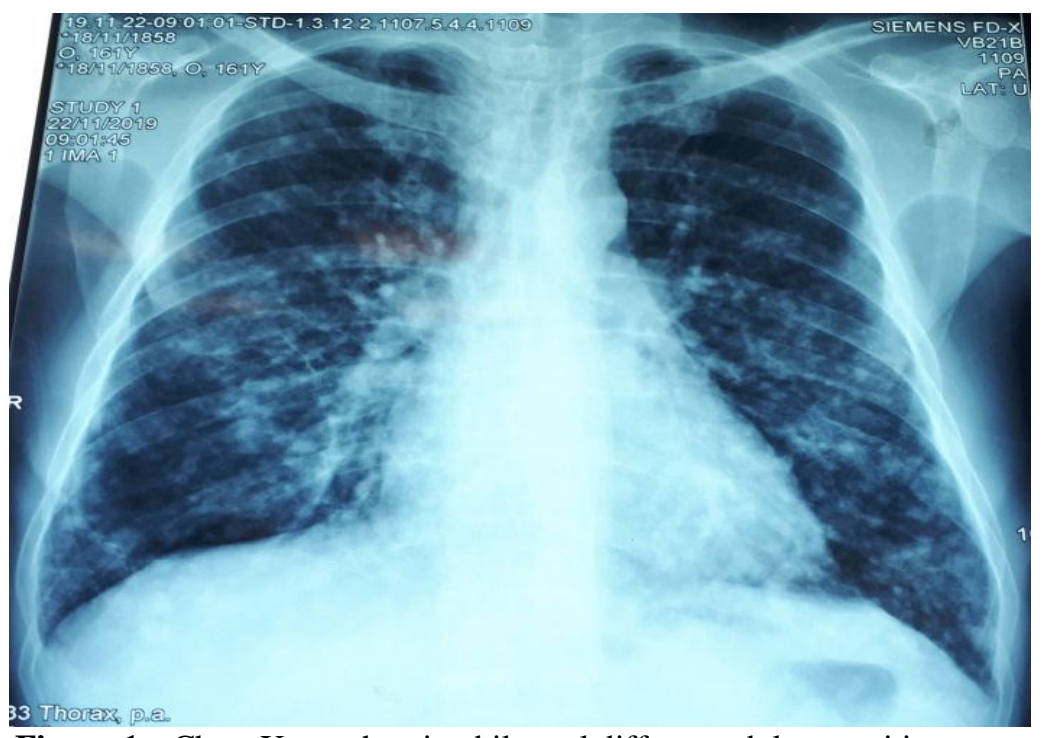

Figure 1:- Chest X-ray showing bilateral diffuse nodular opacities.

A severe pancytopenia is revealed, but renal function and the calcium level were within normal limits.

Chest computed tomography revealed micronodules and nodules in both lungs (Fig 2)with ostealytic lesions of rachis that were suggestive of interstitial lung disease.

Figure 2:- Chest computed tomography showing micronodules and nodules in both lungs with ostealytic lesions of rachis

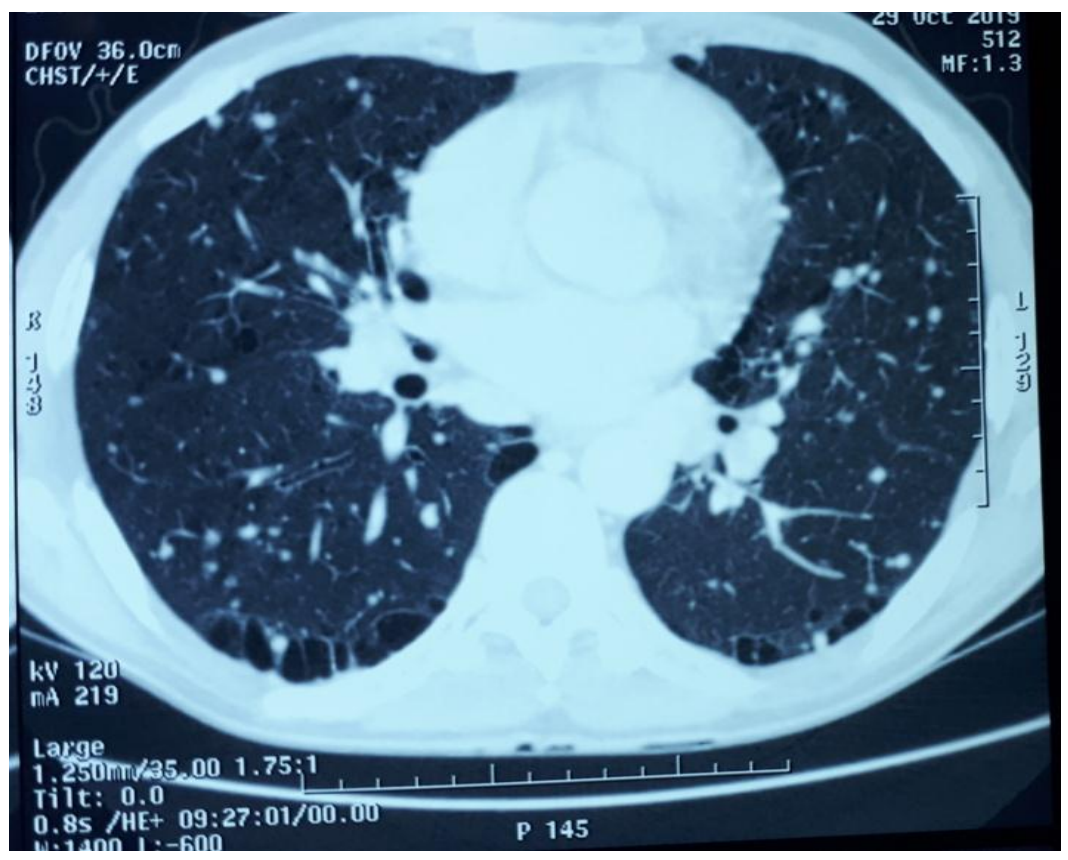

So the next step was bronchoscopy that reveal an Inflammation of the bronchial mucosa with stenosis of apical segment of the right upper lobe (Fig 3); bronchial biopsies, stenosis's biopsies were made. 
Figure 3:- Stenosis of apical segment of the right upper lobe in bronchoscopy

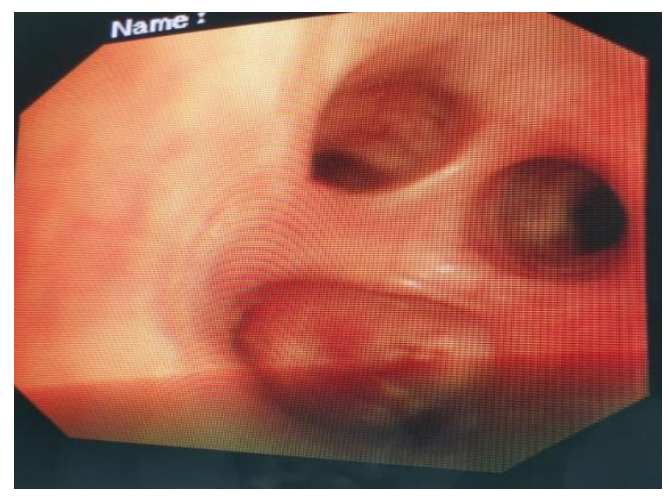

Bronchoalveolar lavage (BAL) fluid was examined; microscopic examination smear / cytospin showed the presence of monoclonal plasmatic cells in BAL (Fig 4,5).
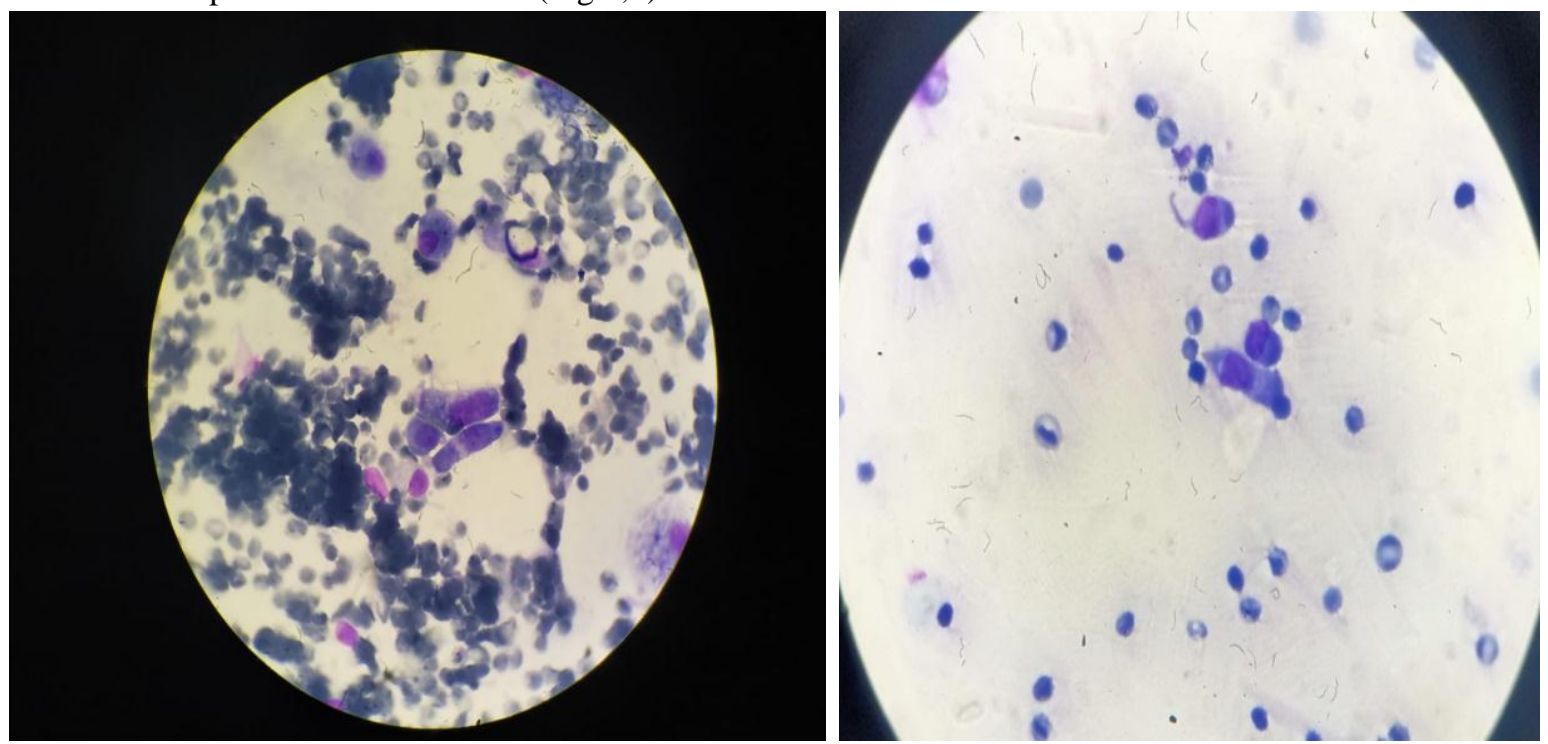

Figure 4,5:- Microscopic examination of BAL smear/ cytospin showing the presence of plasma cells

The patient was transferred to the departement of hematology where he was further investigated and treated for his severe pancytopenia.

He was diagnosed with Multiple myeloma IgA

\section{Discussion:-}

Extramedullary dissemination involves the spleen, liver, lymph nodes, thyroid, adrenal, ovary, testis, lung, pleura, pericardium, gastrointestinal tract and skin; it can be seen in an estimated $13 \%$ of patients; pulmonary spread is extremely rare; While pulmonary findings in $\mathrm{MM}$ are relatively common, the over-whelming majority of these are related to an infectious etiology [2]; a search of the literature revealed three prior cases of multiple pulmonary nodules occurring in multiple myeloma. Other manifestations of pulmonary involvement in multiple myeloma include pneumonia, mass lesion, interstitial disease and calcification. Review of the literature revealed seven case reports of patients with MM found to have interstitial pulmonary infiltration with neoplastic plasma cells, of which two were the initial presentation of myeloma and five were in patients previously diagnosed and treated [3-4]

The diagnosis of extramedullary dissemination in the lungs can be determined by bronchoalveolar lavage (monclonal plasma cells are found) or lung biopsy (interstitial infiltrate of plasma cells)[7].

Our patient's BAL results revealed monoclonal plasma cells on microscopic examination smear / cytospin. This approach to diagnosis is significant given that a diagnosis of MM causing ILD can be challenging to confirm; only 
isolated cases with histological proofs have been reported in the literature. In the majority of cases reviewed, the diagnosis was either made via biopsy (trans-bronchial or surgical) or at autopsy [6-4]. However, two cases were reported in the literature of BAL flow cytometry revealing monoclonal plasma cells in patients who had histologically proven myelomatous involvement of the pulmonary parenchyma $[3, \underline{5}]$. These two cases, along with the case we have presented, suggest that evaluation of BAL samples could help establish the diagnosis and avoid the need for more invasive biopsy techniques.

\section{Conclusion:-}

We present a case of pulmonary infiltration revealing multiple myeloma by bronchoalveolar lavage.

Diffuse parenchymal infiltrates in the lung due to $\mathrm{MM}$ are rare but should be considered when finding pulmonary infiltrates.

\section{Consent:}

Written informed consent was obtained from the patient for publication of this case report and any accompanying images.

\section{Disclosure:}

This case report was written based on clinical observation without any funding.

\section{Conflicts of interest:}

There are no conflicts of interest between the authors and between the authors and the patient.

\section{Data availability statement :}

I agree to share all the data

\section{References:-}

1. C. Touzeau, P. Moreau, How I treat extramedullary myeloma, Blood 127 (8)2015971-976

2. J.S. Kintzer, E.C. Rosenow, R.A. Kyle, Thoracic and pulmonary abnormalities inmultiple myeloma. A review of 958 cases, Arch. Intern. Med. 138 (5) 1978727-730

3. Y. Yuan, R. Wieczorek, D.L. Green, P. Cook, H. Ballard, D.J. Araten, Multiplemyeloma involving skin and pulmonary parenchyma after autologous stem celltransplantation, J. Hematol. Oncol. 2 (1) 200948

4. A.T.W. Ma, R.C.L. Lo, Y. Cheng, C.S. Chim, Multiple myeloma presenting as inter-stitial lung disease, J. Clin. Oncol. 29 (6) 2011143-144

5. Ravinet, S. Perbet, R. Guieze, et al., Lung postmortem autopsy revealing extra-medullary involvement in multiple myeloma causing acute respiratory distresssyndrome, Case Rep. Hematol. 2014 20141-3

6. R. Kamble, T. Rosenzweig, Diffuse pulmonary parenchymal involvement in multiplemyeloma: antemortem diagnosis, Int. J. Hematol. 83 (3) 2006259-261

7. Kamble R, Rosenzweig T. Diffuse pulmonary parenchymal involvement in multiple myeloma: antemortem diagnosis, Int J Hematol. 2006 Apr;83(3):259-61. 